\title{
Experimental verification of Förster energy transfer and quantum resonance between semiconductor quantum dots
}

DaeGwi Kim, TaeGi Lee, Yong-Shin Lee, Taichi Watanabe

\begin{tabular}{|c|l|}
\hline Citation & Current Applied Physics, Volume 18, Supplement, Pages S14-S20 \\
\hline Issue Date & $2018-08$ \\
\hline Type & Journal Article \\
\hline Textversion & Author \\
\hline Right & $\begin{array}{l}\text { C } 2017 \text { Elsevier B.V. This manuscript version is made available under the } \\
\text { This is accepted manuscript version. Please cite only the published version. The } \\
\text { article has been published in final form at https://doi.org/10.1016/j.cap.2017.11.012 }\end{array}$ \\
\hline DOI & \begin{tabular}{l}
$10.1016 /$ j.cap.2017.11.012 \\
\hline
\end{tabular} \\
\hline
\end{tabular}

Self-Archiving by Author(s)

Placed on: Osaka City University Repository

KIM, D., LEE, T., LEE, Y.-S., \& WATANABE, T. (2018). Experimental verification of Förster energy transfer and quantum resonance between semiconductor quantum dots. Current Applied Physics: Supplement. 18, S14-S20. doi:10.1016/j.cap.2017.11.012 
Experimental verification of Förster energy transfer and quantum resonance between semiconductor quantum dots

DaeGwi Kim*, TaeGi Lee, Yong-Shin Lee, and Taichi Watanabe

Department of Applied Physics, Graduate School of Engineering, Osaka City University,

\title{
3-3-138, Sugimoto, Sumiyoshi-ku, Osaka 558-8585, Japan
}

E-mail: tegi@a-phys.eng.osaka-cu.ac.jp

\begin{abstract}
$\underline{\text { Abstract }}$
Quantum dot (QD) superlattices have the potential to provide new optical properties and functions based on interactions between adjacent QDs. Two types of interactions occur between the QDs: energy transfer (ET) from small-sized QDs to large QDs and resonant coupling between QDs with equal eigenenergies. Since ET and resonant coupling strongly depend on the distance between QDs, it is critical to precisely control the distance to understand the interaction mechanism. In this review, we describe that the distance between QDs can be controlled with an accuracy of $1 \mathrm{~nm}$ by a layer-by-layer method and further explain the mechanisms of ET and resonant coupling between adjacent QDs.
\end{abstract}

Keywords: Quantum Dots, Superlattice, Energy Transfer, Quantum Resonance, Layer-by-layer Assembly 


\section{$\underline{\text { Introduction }}$}

In semiconductor quantum dots (QDs) with sizes smaller than $10 \mathrm{~nm}$, quantum effects arise because of the finite size of the QDs [1,2]. As a result, the QDs exhibit physical/chemical properties and functions different from those of the bulk crystal [3-6]. Thus, semiconductor QDs are considered as a promising new functional material. Notable properties of semiconductor QDs include the ability to control the photoluminescence (PL) energy by changing the QD size and high PL efficiency [7-12]. Since the optical properties of semiconductor QDs depend on their size, it is extremely important to prepare QDs with uniform size. Furthermore, since the surface-to-volume ratios of QDs are much larger than those of bulk crystals, the surface structure greatly affects the emission characteristics of the QDs [7, 8]. Thus, controlling both the size and surface structure of QDs is critical.

Most techniques for preparing QDs can be categorized as physical methods involving molecular beam epitaxy $[13,14]$ or chemical syntheses of colloidal QDs using chemical reactions in solution $[7-12,15,16]$. The PL efficiency of QDs can be enhanced by preparing the QDs with core/shell structures [7, 8, 12]. To date, high-quality, size-tunable, and monodispersed colloidal QDs have been demonstrated.

While randomly dispersed QDs have been the focus of most past studies, QD super lattices, in which the QDs are regularly arranged, have the potential to provide new optical properties and functions based on the interactions between adjacent QDs [17-19]. Two types of interactions occur between adjacent QDs: excitation energy transfer (ET) from small-sized QDs to large QDs and resonant interactions between QDs with equal eigenenergies. In ref. [20], Klimov and colleagues reported on resonance ET between CdSe QDs. This inspired studies on QD-based ET processes [21, 22]. ET between QDs 
has generally been attributed to the Förster mechanism [20-22], which is responsible for ET between organic molecules [23]. However, the appropriateness of the Förster model for explaining ET between QDs. The question remains. However, the appropriateness of the Förster model for explanation of ET between QDs has not been verified. Furthermore, although many studies on ET between QDs have been reported, reports on resonance interactions between QDs are limited.

Since ET and resonant interactions strongly depend on the distance between QDs, it is critical to precisely control the distance between QDs to clarify their interaction mechanism. The layer-by-layer (LBL) method can be used to control the distance between QDs [24-26] because (1) it is possible to laminate QDs dispersed in an aqueous solution on a substrate layer by layer, and (2) the interlayer distance can be controlled by the thickness of the spacer (electrolyte polymer) layer. Multilayered structures of semiconductor QDs such as CdTe [21, 27], CdSe [28, 29], and CdS [30, 31] have been fabricated via LBL assembly.

In this review, we describe the precise control of interlayer distance in QD layered structures using the LBL method and discuss the mechanisms of ET and resonant interaction between QDs with a focus on the authors' research results. CdS and CdTe QDs were the focus of studies on ET and resonant interaction, respectively.

\section{Synthesis and optical properties of CdS QDs}

CdS QDs can be synthesized by injecting $\mathrm{H}_{2} \mathrm{~S}$ gas $(0.2 \mathrm{mmol})$ into $100 \mathrm{ml}$ of an aqueous solution containing $0.2 \mathrm{mmol} \quad \mathrm{Cd}\left(\mathrm{ClO}_{4}\right)_{2}$ and $0.2 \mathrm{mmol}$ sodium hexametaphosphate, a dispersion agent for colloids [32]. Figure 1(a) shows the absorption and PL spectra of as-grown CdS QDs. In the absorption spectrum, the 
absorption structure is observed on the high-energy side of the band gap energy of $\sim 2.5$ $\mathrm{eV}$ in the bulk crystal, but the spectrum is very broad because of the wide size distribution of the QDs. In the PL spectrum, the defect-related PL band is the main PL band, and band-edge PL is very weak. These spectra are typical of conventional CdS QDs. In ref. [33], Peng and colleagues have reported the synthesis of CdS QDs whose size is uniform and band-edge PL is observed as the main PL band by the hot-injection method.

The wide size distribution can be remedied using size-selective photoetching [33, 34]. When CdS is irradiated with light under dissolved oxygen, electrons and holes are developed, resulting in the dissolution of $\mathrm{CdS}$ via the following chemical reactions in the vicinity of the surface [34].

$$
\begin{aligned}
& \mathrm{CdS} \rightarrow \mathrm{CdS}\left(\mathrm{nh}^{+}, \mathrm{ne}^{-}\right) \\
& \mathrm{CdS}\left(\mathrm{nh}^{+}, \mathrm{ne}^{-}\right)+2 \mathrm{O}_{2} \rightarrow \mathrm{Cd}^{2+}+\mathrm{SO}_{4}^{2-}
\end{aligned}
$$

Specifically, first, a sample is irradiated with monochromatic light corresponding to the absorption edge. Among the QDs of different sizes, the QDs whose exciton energies are resonant with the irradiation-light energy are photoetched. As the exciton energy of the QD increases with a decrease in the QD size, the QDs to be photoetched become smaller by increasing the irradiation-light energy. This process results in a narrow size distribution, and the QDs with uniform size can be finally obtained [33, 34]. The photoetching method is very simple, it only irradiates the CdS QD solution with monochromatic light combining Xe lamp and interference filter.

Figure 1(b) shows the absorption spectrum of CdS QDs after size-selective photoetching. Photoetching narrowed the QD size distribution, causing a clear 
absorption peak to appear in the absorption spectrum. The average diameter of the QDs and the width of the size distribution were estimated from the absorption spectrum via line-shape analysis to be $4.5 \mathrm{~nm}$ and $5 \%$, respectively [35].

Figure 1(c) shows the absorption and PL spectra of surface-modified CdS QDs after photoetching. The $\mathrm{pH}$ of the sample solution after photoetching was adjusted to the alkaline range, and an aqueous solution of cadmium ions was added to modify the surface. As a result, the surfaces of the CdS QDs were covered with $\mathrm{Cd}(\mathrm{OH})_{2}$ layers $[34,36]$, and band-edge PL was observed as the main PL band. Furthermore, the QD size could be controlled by the wavelength of the light used for irradiation during photoetching. CdS QDs with mean diameters of 4.5 and $5.0 \mathrm{~nm}$ were used as energy donors (D) and acceptors (A), respectively.

\section{$\underline{\text { Synthesis and optical properties of CdTe QDs }}$}

CdTe QDs can be synthesized by a hydrothermal method as described in the previous paper $[11,37]$. Briefly, freshly prepared NaHTe solution was injected into the solutions of $\mathrm{Cd}\left(\mathrm{ClO}_{4}\right)_{2} \cdot 6 \mathrm{H}_{2} \mathrm{O}$ and $N$-acetyl- $L$-cysteine (NAC) at $\mathrm{pH}$ 7.0. A molar ratio and concentration of monomers of $\mathrm{Cd}: \mathrm{Te}: \mathrm{NAC}=1.0: 0.2: 1.2$ and $\left[\mathrm{Cd}^{2+}\right]=20 \mathrm{mM}$ were used. Then, $10 \mathrm{~mL}$ of the solution was loaded into an autoclave and incubated at $200{ }^{\circ} \mathrm{C}$ for a specified period. The QD size can be controlled by the reaction time as discussed in refs. [11] and [37].

Figure 2 shows the absorption and PL spectra of the CdTe QDs prepared at $200{ }^{\circ} \mathrm{C}$ for various reaction times. As reaction time increased, the absorption peak energy shifted toward the lower-energy side. Thus, the mean size of the CdTe QDs could be controlled by changing the reaction time. The lowest exciton energy originated from the 
$1 \mathrm{~s}-1 \mathrm{~s}$ electronic transition in CdTe QDs, where the diameter $d$ is given by [38]

$$
E(d)=1.596+\frac{1}{0.137 d^{2}+0.206} \quad(\mathrm{eV})
$$

Using Eq. (1), the mean diameters were estimated from the absorption peak energy to be 2.4, 2.6, 2.8, 3.3, and $3.5 \mathrm{~nm}$ for CdTe QDs synthesized with reaction times of 3, 5, 10, 20, and $30 \mathrm{~min}$, respectively. In the PL spectra, the band-edge PL is dominant, and the defect-related PL is negligibly weak.

Preparation of bilayer structures of CdS D-and A-QDs and multilayer structures of CdTe QDs by LBL assembly

LBL is a simple and powerful technique for preparing multilayer structures by alternately adsorbing positively and negatively charged species using electrostatic interactions [24-26]. A highly homogeneous QD/polymer multilayer can be made by combining negatively charged semiconductor QDs with a positively charged cationic polymer such as poly(diallyldimethylammonium chloride) (PDDA) by LBL assembly. The distance between QD layers can be controlled by the spacer-layer thickness by using LBL assembly comprising positively charged polyelectrolytes of PDDA and negatively charged poly(acrylic acid) (PAA). The thickness of the spacer layer was controlled by the number of PAA/PDDA bilayers, and the average thickness of one bilayer of PAA/PDDA was estimated to be $0.9 \mathrm{~nm}$ by spectroscopic ellipsometry measurement [39]. Thus, the distance between QD layers in the bilayer structure can be controlled within an accuracy of $0.9 \mathrm{~nm}$.

To clarify the ET mechanism, the bilayer structures of CdS D-QDs and A-QDs were prepared. The bilayer structures were composed of three main blocks: (1) a monolayer of A-QDs; (2) a spacer layer of PDDA/(PAA/PDDA $)_{n}$, where (PAA/PDDA) ${ }_{n}$ indicates $n$ 
bilayers of PAA and PDDA; and (3) a monolayer of D-QDs. Thus, the bilayer structure is given as $\mathrm{A}-\mathrm{QDs} /\left[\mathrm{PDDA} /(\mathrm{PAA} / \mathrm{PDDA})_{n}\right] / \mathrm{D}-\mathrm{QDs}[39]$.

QD multilayer structures of CdTe QDs were prepared to study resonant interaction by the LBL assembly of negatively charged CdTe QDs and positively charged PDDA. The resonance interaction between adjacent QDs was observed by laminating CdTe QDs of the same size using the LBL method, and the interaction mechanism was clarified from the dependence of interaction energy on the interlayer distance.

\section{$\underline{\text { ET mechanism }}$}

As shown in Fig. 1, the absorption spectrum of A-QDs (average diameter of $5.0 \mathrm{~nm}$ ) and the emission spectrum of D-QDs (average diameter $4.5 \mathrm{~nm}$ ) have sufficient overlap. Thus, ET from D-QDs to A-QDs will occur by decreasing the distances between these QDs. Figure 3(a) shows the PL decay profile in a reference sample containing only D-QDs dispersed in a polyvinyl alcohol (PVA) film. In order to eliminate the influence of nonradiative processes, the measurements were performed at low temperature of 10 K. As in previous reports $[40,41]$, the PL decay profile shows non-exponential decay. No method for the quantitative analysis of such a non-exponential PL decay profile has been established yet. The decay profile of CdS QDs can be well reproduced by combining a single exponential function with a stretched exponential function as follows [34].

$$
I(t)=A_{1} \exp \left(-t / \tau_{1}\right)+A_{2} \exp \left[-\left(t / \tau_{2}\right)^{\beta}\right]
$$

In Eq. (2), the single exponential component $\tau_{1}$ represents the decay time of the optically allowed bright exciton state [|Br>; Fig. 3(b)]. The temperature dependence of the stretched exponential component $\tau_{2}$ is explained by a three-state model consisting of 
a ground state and two excited states: a lower-lying bound-exciton state $(\mid \mathrm{Bx}>)$ and a higher-lying optically forbidden dark exciton state $(\mid \mathrm{Dx}>)$ [34]. One example of the analysis is shown in Fig. 3. The PL decay profiles are reasonably explained by the fitting model in the entire time range.

Figure 3(c) shows the PL decay profiles of the D-QDs in the bilayer structure of A-QDs/PDDA/D-QDs and in the PVA film at $10 \mathrm{~K}$. In order to eliminate the contribution of in-plane ET process in the D-QD layer, the PL decay profiles were detected at $2.64 \mathrm{eV}$ that is lower than the absorption onset energy. The decay profile detected at $2.64 \mathrm{eV}$ in the monolayer of the D-QDs corresponds to that in the PVA film, indicating suppression of the in-plane ET process. The decay profile in the bilayer structure is faster than that in the PVA film, clearly demonstrating that ET from the D-QDs to the A-QDs occurs in the bilayer structure. The broken curves denote the results of the fitting analysis using Eq. (2). A notable difference in $\tau_{1}$ is observed (20 ns in the PVA film and $7 \mathrm{~ns}$ in the bilayer structure). The ET rate, $1 / \tau_{\mathrm{ET}}$, was calculated to be $0.09 \mathrm{~ns}^{-1}$ by the difference between the recombination rate in the bilayer structure and in the PVA film sample:

$$
\frac{1}{\tau_{\mathrm{ET}}}=\frac{1}{\tau_{\text {bilayer }}}-\frac{1}{\tau_{\mathrm{PVA}}}
$$

where, $1 / \tau_{\text {bilayer }}$ is the recombination rate in the bilayer sample, and $1 / \tau_{\mathrm{PVA}}$ is the recombination rate in the PVA film sample.

Figure 4 shows the PL decay profiles of the D-QDs in the bilayer structures of A-QDs/[PDDA/(PAA/PDDA $\left.)_{n}\right] / \mathrm{D}-\mathrm{QDs}$ with different numbers of PAA/PDDA bilayers. As the number of PAA/PDDA bilayers increased (i.e., the thickness of the spacer layer increased), the PL decay profile of the D-QDs gradually became longer. At $n=7$, the decay profiles in the bilayer structure and PVA film were similar, indicating full 
suppression of the ET process.

Figure 5 shows the dependence of the ET rate $\left(1 / \tau_{\mathrm{ET}}\right)$ on the distance between the D-QDs and A-QDs, which is defined as $d=R_{\mathrm{D}}+L+R_{\mathrm{A}}$, where $R_{\mathrm{D}}$ and $R_{\mathrm{A}}$ denote the radii of the D-QDs and A-QDs, respectively, and $L$ is the thickness of the spacer layer. Least-squares analysis (indicated by the solid line in Fig. 5) indicated that the ET rate between CdS QDs is inversely proportional to the $4^{\text {th }}$ power of $d$.

Based on the Förster model, the ET rate from energy donor to acceptor is given by $[20,21,23]$

$$
k_{E T}=\frac{2 \pi}{\hbar} \times \frac{\mu_{D}^{2} \mu_{A}^{2} \kappa^{2}}{R^{6} n^{4}} \int d E L(E) \cdot A(E),(4)
$$

where $\mu_{\mathrm{D}}$ and $\mu_{\mathrm{A}}$ denote the transition dipole moment of the energy donor and acceptor, respectively; $\kappa^{2}$ is the dipole orientation factor; $n$ is the refractive index of the medium; and $R$ is the distance between two dipoles of the donor and acceptor. Thus, the ET rate is inversely proportional to the 6th power of $R$. This $1 / R^{6}$ dependence is different from the $1 / d^{4}$ dependence shown in Fig. 5. The Förster model [Eq. (4)] is related to ET between two isolated dipoles; however, the D-QDs and A-QDs in the bilayer structure have a layered structure. Therefore, it is necessary to consider ET from one D-QD to all the A-QDs in the layered structure of the acceptor layer. Assuming that the surface density of QDs in the A-QD layer is $\sigma$, the total ET rate is given by the surface integral:

$$
k_{E T} \propto \sigma \int \frac{1}{R^{6}} d S .
$$

As discussed in ref. [39], by performing the surface integral of eq. (5), the $d$-dependence of ET from D-QDs in the bilayer structure is given by

$$
k_{E T} \propto \frac{1}{d^{4}} .
$$

Therefore, the observed relationship between ET rate in the bilayer structure to $d$ can be 
explained by Förster ET between the D-QDs and A-QDs in the layered structure. In other words, it was experimentally verified that ET between semiconductor QDs is based on the Förster mechanism.

\section{Experimental observation of resonant interaction}

Semiconductor QD superlattices, in which QDs are periodically ordered, represent an exciting new class of photovoltaic materials. These "artificial solids" are expected to exhibit novel properties arising from the resonant interactions between adjacent QDs. Recently, three-dimensional (3D) superlattices of CdTe QDs were successfully prepared from LBL assemblies of positively charged PDDA and negatively charged CdTe QDs [42].

The formation of ordered arrays of CdTe QDs has been confirmed by X-ray-diffraction (XRD). Figures 6(a) and (b) shows out-of-plane and in-plane XRD patterns, respectively, of a multilayer structure of (PDDA/CdTe $)_{10}$ fabricated using CdTe QDs with diameters of $3.3 \mathrm{~nm}$. Diffraction peaks are observed at $2.3^{\circ}$ in Fig. 6(a) and at $1.9^{\circ}$ in Fig. 6(b), demonstrating that the CdTe QD multilayers have a structural regularity with a periodicity of $3.9 \mathrm{~nm}$ in the lamination direction and $4.7 \mathrm{~nm}$ in the in-plane direction. The larger periodicity in the in-plane direction is attributed to the effect of electrostatic repulsion between negatively charged CdTe QDs. The results in Fig. 6 indicate that periodically-ordered 3D superlattices of CdTe QDs can be deposited using LBL assemblies.

Figure 7(a) shows the absorption spectra of CdTe QD layered structures with different numbers of (PDDA/CdTe $)_{n}$ bilayers. As the layer number $n$ increases, two characteristic behaviors are observed: an increase in absorption intensity and a 
low-energy shift in absorption peak energy. The dependence of optical density at the absorption peak on $n$ is shown in Fig. 7(b). The absorption intensity increases linearly with $n$, indicating that the CdTe QDs are adsorbed uniformly during LBL deposition. This is one merit of LBL assemblies for preparing homogeneous 3D QD superlattices.

Figure 8(a) shows the absorption peak energy as a function of $n$. The absorption peak energy shifts to lower energy with increasing $n$. This shift toward lower energy was attributed to resonant interaction between adjacent QDs [42]. The resonant interaction lowers the intrinsic energy by the coupling energy between adjacent QDs, thereby red-shifting the absorption peak. As the physical origin of the resonant interaction, two mechanisms have been considered [17, 42, 43]: dipole-dipole coupling and quantum resonance. The dipole-dipole coupling is a long-range interaction, and its strength is inversely proportional to the $6^{\text {th }}$ power of the separation distance between the two dipoles (i.e., the center-to-center distance between QDs). On the contrary, the quantum resonance is based on overlapping of wave functions between adjacent QDs due to the tunneling effect. Thus, it is a short-range interaction for which the interaction energy depends exponentially on the surface-to-surface distance between QDs. Therefore, by measuring the dependence of the coupling energy on the inter-QD distance, the responsible mechanism can be determined.

Figure 8(b) shows the dependence of the energy shift between the absorption peaks of the CdTe QD monolayer and the CdTe QD bilayer $\left(\mathrm{CdTe} / \mathrm{PDDA} /(\mathrm{PAA} / \mathrm{PDDA})_{m} / \mathrm{CdTe}\right)$ on the thickness of the spacer layer, which can be controlled by the layer number $m$ of $(\mathrm{PAA} / \mathrm{PDDA})_{m}$. The energy shift decreases exponentially with spacer layer thickness, indicating that the physical origin of the resonant interaction is quantum resonance between adjacent CdTe QDs in the 
lamination direction.

\section{$\underline{\text { Summary and outlook }}$}

We have presented an overview of the experimental results clarifying the mechanisms of ET and resonant interaction between adjacent QDs with a focus on the authors' research results. A bilayer structure of CdS D-QDs and A-QDs was prepared to investigate the ET process between QDs. The distance between two QD monolayers in the bilayer structure could be controlled with nanometer accuracy by using an LBL assembly of oppositely charged polyelectrolytes. ET between the D-QDs and A-QDs was conclusively determined to be caused by dipole-dipole interactions, which verifies the appropriateness of the Förster model.

XRD structural analysis demonstrated that periodically-ordered 3D superlattices of CdTe QDs can be deposited using LBL assemblies. Resonant interaction between adjacent QDs has been discussed based on experimental absorption properties. Furthermore, based on the dependence of the interaction energy on spacer layer thickness, we demonstrated that the physical origin of the resonant interaction is quantum resonance between adjacent CdTe QDs in the lamination direction.

To understand the PL mechanism in QDs, it is necessary to systematically study the temperature dependence of the PL spectra and PL decay profiles. Similarly, to further understand the ET and resonant interactions, it is important to systematically investigate the absorption and PL characteristics, PL dynamics, and their temperature dependences. To date, most studies on ET between QDs have been conducted at room temperature, whereas little attention has been paid to the temperature dependence of ET. In one study on the temperature dependence of ET between $\mathrm{CdSe} / \mathrm{ZnS}$ QDs, the observed ET rate 
was dominated by the thermal population behavior of the bright exciton state under the condition that the thermal energy is comparable to the splitting energy between the bright and dark exciton states and above [44]. The authors also suggested a breakdown of thermal equilibrium between the bright and dark exciton states in the low-temperature region and proposed that the temperature dependence of the relaxation rate is a key factor in the observed ET rate.

Regarding resonant interaction, most studies have focused on absorption characteristics. The nonradiative processes and/or exciton localization would affect PL properties of QD superlattices. Thus, systematic investigations of both PL characteristics and their temperature dependences will deepen our understanding of the nature of resonant interaction. Moreover, such studies are needed to provide important knowledge for the application of QD superlattices in solar cells [45]. 
References

[1] A. I. Ekimov and A. A. Onushchenko, Sov. Phys. Semiconductors-USSR 16 (1982) $775-778$.

[2] A. Henglein, Ber. Bunsenges. Phys. Chem. 86 (1982) 301-305.

[3] U. Woggon, Optical Properties of Semiconductor Quantum Dots (Springer, 1996).

[4] A. D.Yoffe, Advances in Physics 50 (2001) 1-208.

[5] Y. Masumoto and T. Takagahara, Semiconductor Quantums Dots (Springer, 2002).

[6] V. I. Klimov, Semiconductor and Metal Nanocrystals (Marcel Dekker Inc., 2004).

[7] M. A. Hines and P. Guyot-Sionnest, J. Phys. Chem. B 100 (1996) 468-471.

[8] B. O. Dabbousi, J. Rodriguez-Viejo, F. V. Mikulec, J. R. Heine, H. Mattoussi, R.

Ober, K. F. Jensen, and M. G. Bawendi, J. Phys. Chem. B 101 (1997) 9463-9475.

[9] M. A. Hines and P. Guyot-Sionnest, J. Phys. Chem. B 102 (1998) 3655-3657.

[10] N. Gaponik, D. V. Talapin, A. L. Rogach, K. Hoppe, E. V. Shevchenko, A. Kornowski, A. Eychmüller and H. Weller, J. Phys. Chem. B 106 (2002) 7177-7185.

[11] H. Bu, H. Kikunaga, K. Shimura, K. Takahashi, T. Taniguchi, and D. Kim, Phys. Chem. Chem. Phys. 15 (2013) 2903-2911.

[12] Y.-S. Lee, K. Nakano, H.-B. Bu, and D. Kim, Appl. Phys. Express 10 (2017) 065001 (4 pages). 
[13] P. A. Schultz, K. Leung, and E. B. Stechel, Phys. Rev. B. 59 (1999) 733-741.

[14] P. W. Fry, I. E. Itskevich, D. J. Mowbray, M. S. Skolnick, J. J. Finley, J. A. Barker,

E. P. O'Reilly, L. R. Wilson, I. A. Larkin, P. A. Maksym, M. Hopkinson, M. Al-Khafaji, J. P. R. David, A. G. Cullis, G. Hill, and J. C. Clark, Phys. Rev. Lett. 84 (2000) 733-736.

[15] T. Pons, E. Pic, N. Lequeux, E. Cassette, L. Bezdetnaya, F. Guillemin, F. Marchal, and B. Dubertret, ACS Nano, 4 (2010) 2531-2538.

[16] H. Bu, H. Yokota, K. Shimura, K. Takahasi, T. Taniguchi, and D. Kim, Chem. Lett. 44 (2015) 200-202.

[17] T. Hanrath, J. Vac. Sci. Technol. A 30 (2012) 030802 (28 pages).

[18] J.-H. Choi, A . T. Fafarman, S. J. Oh, D.-K. Ko, D. K. Kim, B. T. Diroll, S. Muramoto, J. G. Gillen, C. B. Murray, and C. R. Kagan, Nano Lett. 12 (2012) 2631-2638.

[19] E. Talgorn, Y. Gao, M. Aerts, L. T. Kunneman, J. M. Schins, T. J. Savenije, M. A. H. Herre, S. J. Zant, A. J. Houtepen, L. D. A. Siebbeles, Nature Nanotech. 6 (2011) 733-739.

[20] S. A. Crooker, J. A. Hollingsworth, S. Tretiak, and V. I. Klimov, Phys. Rev. Lett. 89 (2002) 186802 (4 pages). 
[21] T. Franzl, T. A. Klar, S. Schietinger, A. L. Rogach, and J. Feldmann, Nano Lett. 4 (2004) 1599-1603.

[22] S. F. Wuister, R. Koole, C. M. Donegá, and A. Meijerink, J. Phys. Chem. B 109 (2005) 5504-5508.

[23] T. Förster, Discuss. Faraday Soc. 27 (1959) 7-17.

[24] G. Decher, Science 277 (1997) 1232-1237.

[25] G. Decher and J. B. Schlenoff, Multilayer Thin Films (Wiley-VCH, 2003).

[26] N. A. Kotov, Nanoparticle Assemblies and Superstructures (CRC Press, 2005).

[27] A. A. Mamedov, A. Belov, M. Giersig, N. N. Mamedova, and N. A. Kotov, J. Am. Chem. Soc. 123 (2001) 7738-7739.

[28] Z. Tang, Y. Wang, and N. A. Kotov, Langmuir 18 (2002) 7035-7040.

[29] D. Zimnitsky, C. Jiang, J. Xu, Z. Lin, and V. V. Tsukruk, Langmuir 23 (2007) $4509-4515$.

[30] N. A. Kotov, I. Dékány, and J. H. Fendler, J. Phys. Chem. 99 (1995) 13065-13069.

[31] D. Kim, S. Okahara, K. Shimura, and M. Nakayama, J. Phys. Chem. C 113 (2009) 7015-7018.

[32] D. Kim, T. Mishima, K. Tomihira, and M. Nakayama, J. Phys. Chem. C 112 (2008) 10668-10673. 
[33] W. W. Yu and X. Peng, Angew. Chem. Int. Ed. 41 (2002) 2368-2371.

[34] H. Matsumoto, T. Sakata, H. Mori, and H. Yoneyama, J. Phys. Chem. 100 (1996) 13781-13785.

[35] D. Kim, N. Teratani, and M. Nakayama, Jpn. J. Appl. Phys. 41 (2002) 5064-5068.

[36] L. Spanhel, M. Haase, H. Weller, and A. Henglein, J. Am. Chem. Soc. 109 (1987) $5649-5655$.

[37] T. Watanabe, K. Takahashi, K. Shimura, H. Bu, H. Kim, and D. Kim, Bull. Chem. Soc. Jpn. 90 (2017) 52-58.

[38] C. M. Donegá and R. Koole, J. Phys. Chem. C 113 (2009) 6511-6520.

[39] D. Kim, S. Okahara , M. Nakayama, and Y. Shim, Phys. Rev. B 78 (2008) 153301 (4 pages).

[40] O. Schöps, N. Le Thomas,U. Woggon, and M. V. Artemyev, J. Phys. Chem. 110 (2006) 2074-2079.

[41] G. Mauckner, K. Thonke, T. Baier, T. Walter, and R. Sauer, J. Appl. Phys. 75 (1994) 4167-4170.

[42] D. Kim, S. Tomita, K. Ohshiro, T. Watanabe, T. Sakai, I. Chang, and H. Kim, Nano Letters 15 (2015) 4343-4347.

[43] H. Kim, I. Kim, K. Kyhm, R. A. Taylor, J. S. Kim, J. D. Song, K. C. Je, and L. S. 
Dang, Nano Lett. 16 (2016) 7755-7760.

[44] D. Kim, K. Okazaki, and M. Nakayama, Phys. Rev. B 80 (2009) 045322 (5 pages).

[45] A. J. Nozik, Physica E 14 (2002) 115-120. 


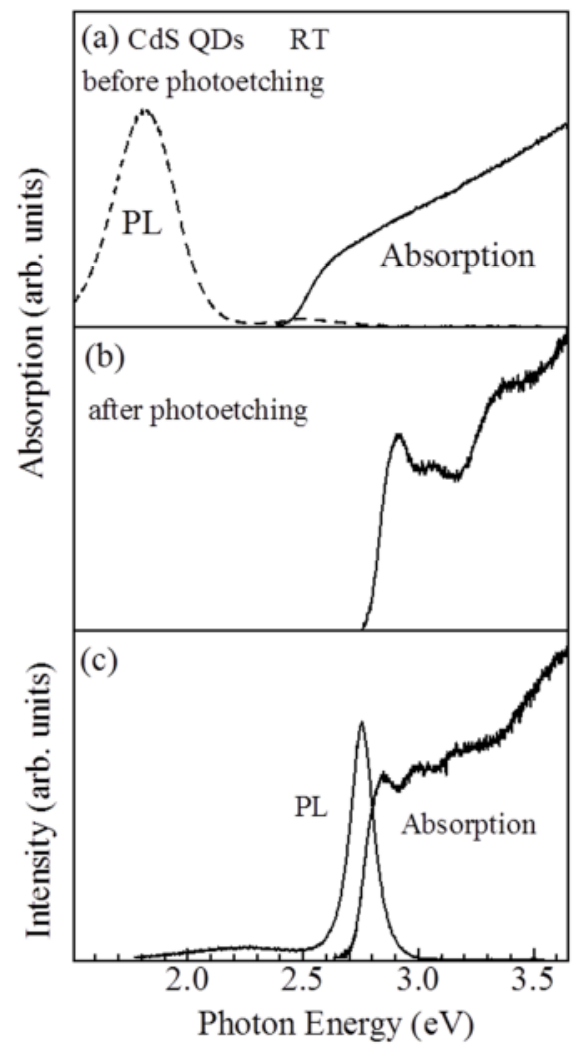

Fig. 1. (a) Absorption and PL spectra of as-grown CdS QDs. (b) Absorption spectrum of CdS QDs after size-selective photoetching. (c) Absorption and PL spectra of surface-modified CdS QDs after photoetching. 


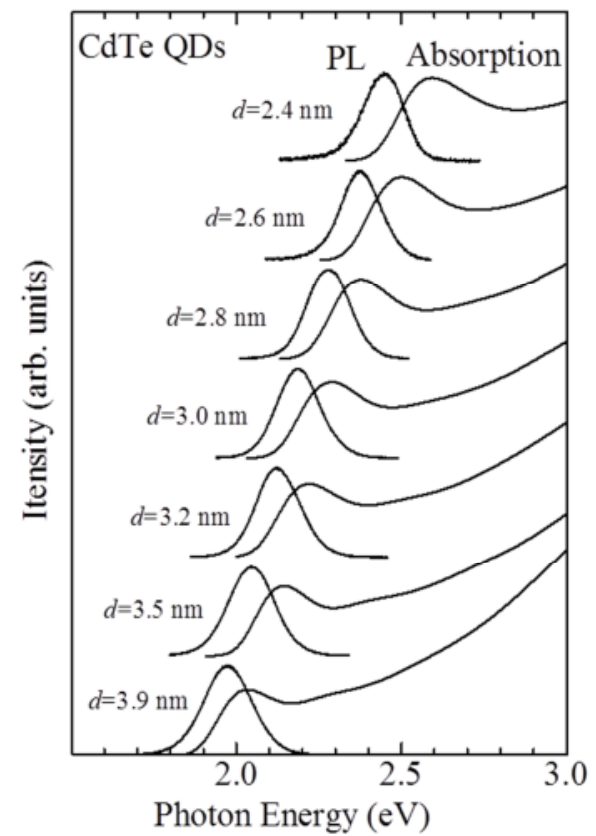

Fig. 2. Absorption and PL spectra of the CdTe QDs prepared at $200{ }^{\circ} \mathrm{C}$ for various reaction times. 

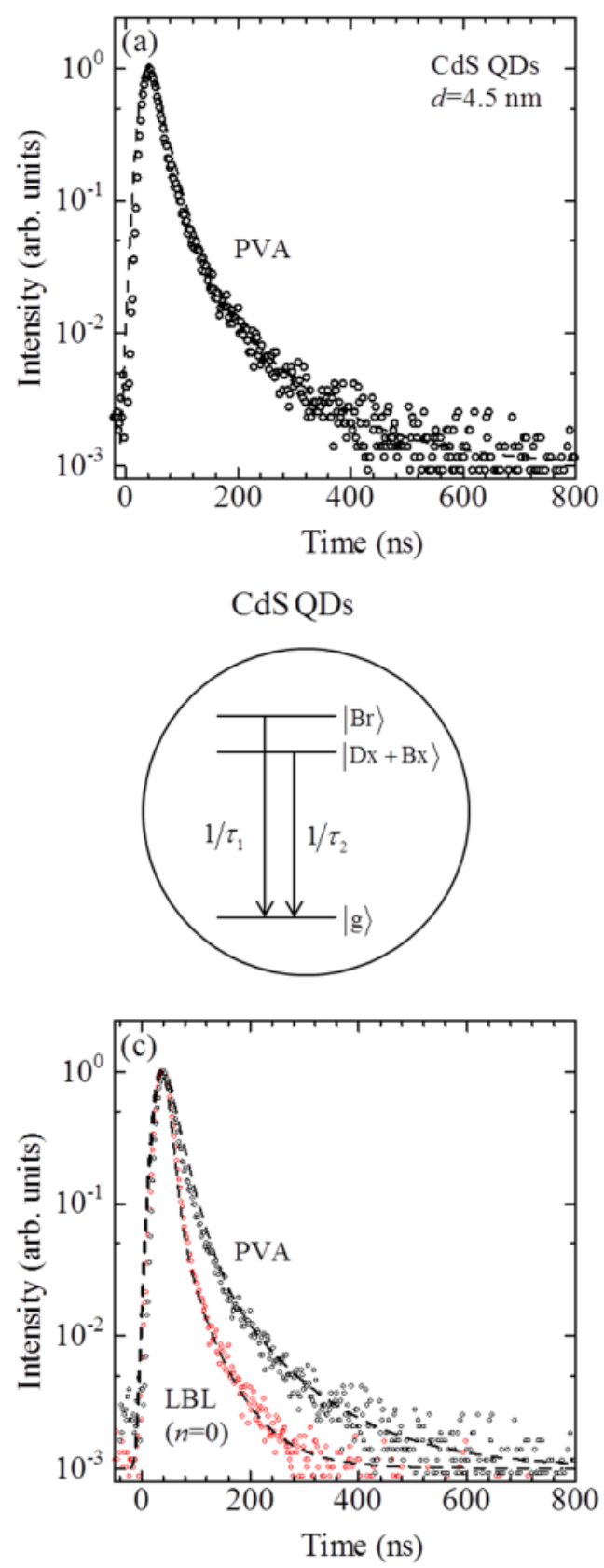
Fig. 3. (a) PL decay profile of the D-QDs dispersed in the PVA film. (b) Schematic of emitting transitions in CdS QDs. (c) PL decay profiles of the D-QDs in the bilayer structure of A-QDs/PDDA/D-QDs and in the PVA film.


Fig. 4. PL decay profiles of the D-QDs in the bilayer structures of A-QDs/[PDDA/(PAA/PDDA $\left.)_{n}\right] / \mathrm{D}-\mathrm{QD}$ with different numbers of PAA/PDDA bilayers. 


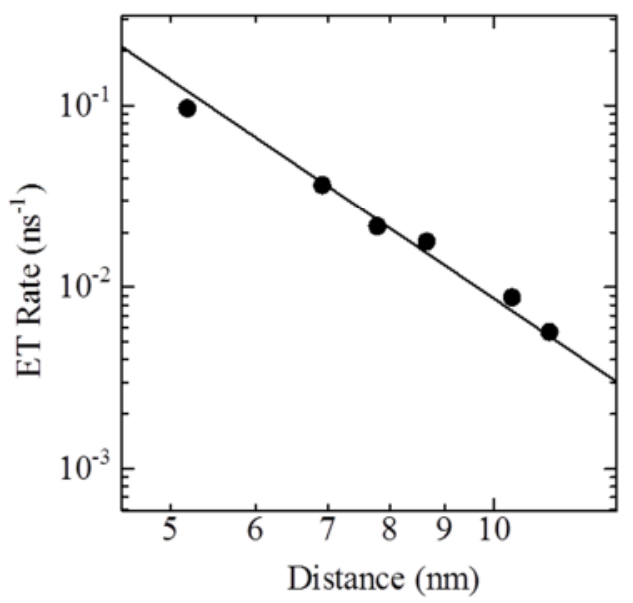

Fig. 5. The dependence of the ET rate on the distance between the D-QDs and A-QDs. 

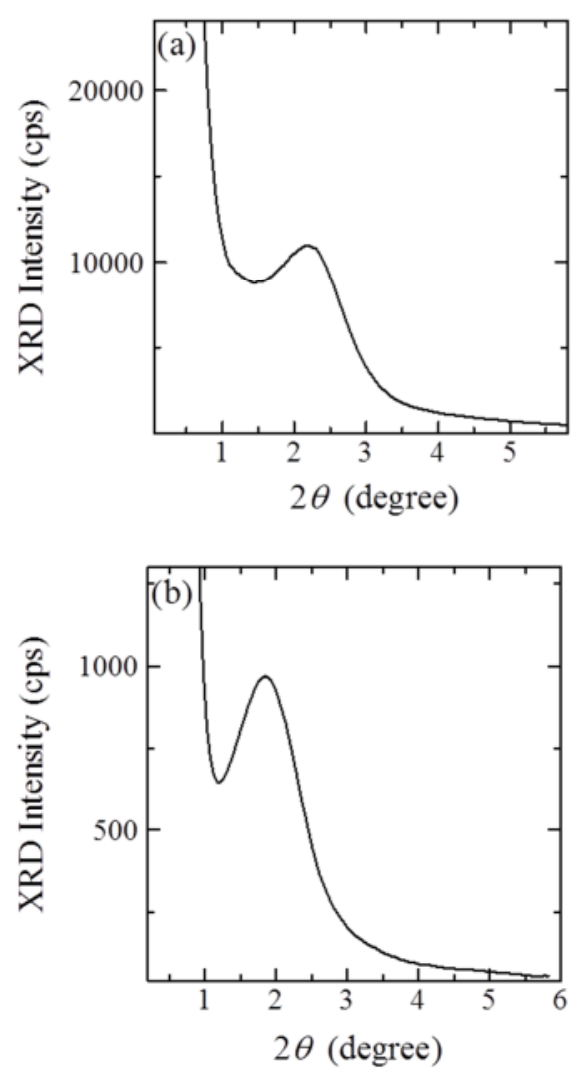

Fig. 6. (a) Out-of-plane and (b) in-plane XRD patterns of a multilayer structure of $(\mathrm{PDDA} / \mathrm{CdTe})_{10}$. 

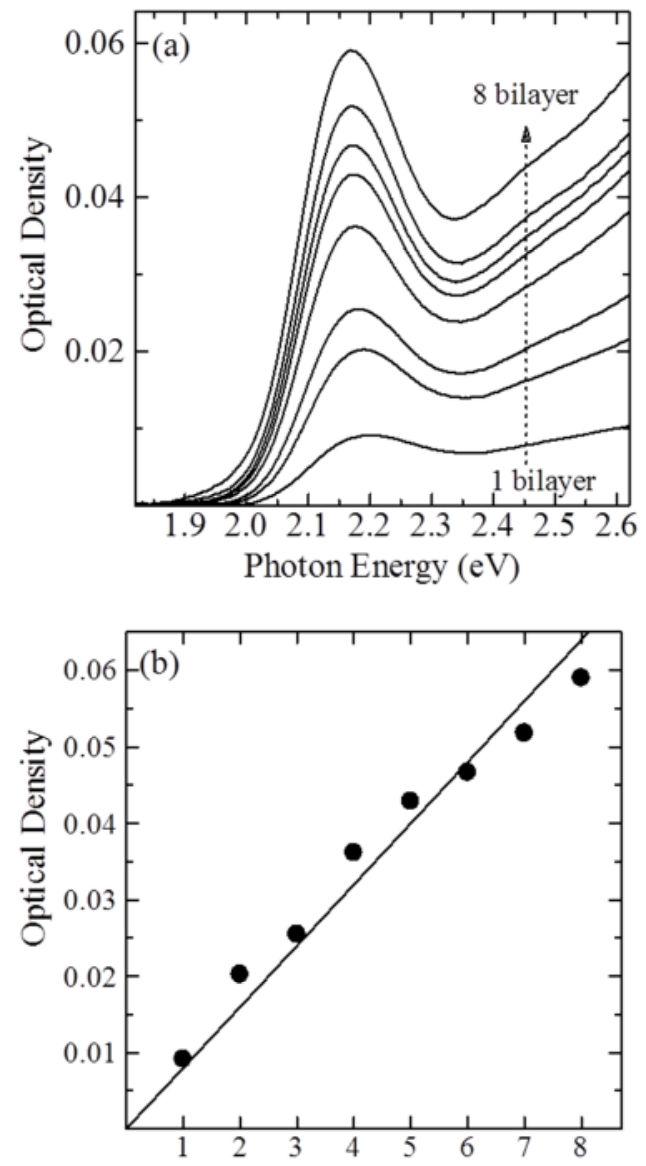

Bilayer number of (PDDA/CdTe-QDs) $n$

Fig. 7. (a) Absorption spectra of CdTe QD-layered structures with different numbers of (PDDA/CdTe) ${ }_{n}$ bilayers. (b) The dependence of optical density at the absorption peak on $n$. 




Bilayer number of (PDDA/CdTe-QDs) $)_{n}$



Fig. 8. (a) The dependence of the absorption peak energy on the layer number $n$. (b) Energy shift between the absorption peak energies of the monolayer and of the CdTe bilayer structure, CdTe-QDs/(PDDA/PAA) $/ \mathrm{PDDA} / \mathrm{CdTe}-\mathrm{QDs}$, as a function of the layer number $m$ of (PDDA/PAA $)_{m}$. 